\title{
A METODOLOGIA Q NAS CIÊNCIAS SOCIAIS E HUMANAS: O RESGATE DA SUBJECTIVIDADE NA INVESTIGAÇÃO EMPÍRICA
}

\author{
Margarida Couto ${ }^{1}$ \\ Carlos Farate ${ }^{2}$ \\ Susana Ramos ${ }^{3}$ \\ Manuela Fleming 4
}

Resumo: No âmbito das ciências sociais e humanas, incluindo a psicologia, não tem sido dada a devida atenção à metodologia $\mathrm{Q}$. Trata-sede uma metodologia com procedimentos apropriados ao estudo de conceitos subjectivos como crenças, atitudes, comportamentos e opiniões e cuja eficácia implica uma grande habilidade e rigor por parte do investigador. Os participantes Q são cuidadosamente seleccionados de forma a constituírem uma amostra de participantes relevantes para a discussão do tema em questão. A análise e tratamento de dados é um processo objectivo e quantitativo, que auxilia a clarificação de alguns dos conceitos estudados no campo das ciências sociais e do comportamento, ao mesmo tempo que integra as metodologias quantitativa e qualitativa numa relação de complementaridade.

Palavras-chave: metodologia $\mathrm{Q}$, subjectividade, investigação empírica, comportamentos humanos.

The $Q$ methodology in the social and human sciences: the release of subjectivity in the empirical research (Abstract): The Q methodology has not yet been given the proper treatment by the Social and Human Sciences, Psychology included. It is a methodology whose procedures are suitable to the empirical study of subjective

\footnotetext{
${ }^{1}$ Margarida Couto (mmaria09@gmail.com) - Instituto de Ciências Biomédicas Abel Salazar - Universidade do Porto. Instituto Superior Miguel Torga.

${ }^{2}$ Carlos Farate (carlos.farate@sapo.pt) - Instituto de Ciências Biomédicas Abel Salazar Universidade do Porto. Instituto Superior Miguel Torga.

3 Susana Ramos (susanaramos@fcdef.uc.pt) - Faculdade de Ciências do Desporto e Educação Física - Universidade de Coimbra. Instituto Superior Miguel Torga.

${ }^{4}$ Manuela Fleming (manuelafleming@iol.pt) - Instituto de Ciências Biomédicas Abel Salazar - Universidade do Porto.
} 
concepts such as beliefs, attitudes, behaviours, and opinions and whose efficacy requires great skill and accuracy by the researcher. Q Participants are carefully selected so as to constitute a relevant sample to the discussion of the topic at issue. The data analysis and processing is an objective and quantitative process, which helps to clarify some of the concepts studied in the social and behavioural sciences. At the same time, it incorporates the quantitative and qualitative methodologies in a complementary relationship.

Keywords: Q-method, subjectivity, empirical studies, human behaviour.

\section{Introdução}

A Metodologia Q foi criada na década de 30 do século passado ${ }^{5}$ por William Stephenson, físico e psicólogo inglês interessado em encontrar recursos para estudar a subjectividade, característica de alguns objectos de estudo presentes em diferentes áreas e contextos, incluindo os contextos pessoal e vivencial. Esta metodologia foi posteriormente desenvolvida nos EUA por Steven Brown, com um aperfeiçoamento significativo no que respeita à investigação empírica nas áreas das ciências sociais e humanas (Watts \& Stenner, 2005a, 2005b).

Trata-se de uma metodologia baseada originariamente numa perspectiva construtivista, segundo a qual os seres humanos agem de acordo com as representações que constroem da realidade, e não de acordo com a própria realidade em si. Este pressuposto implica que a representação da realidade externa seja construída internamente, já que resulta de uma interacção com o meio que é única para cada indivíduo (Boros, Visu-Petra, \& Cheie, 2007).

De acordo com Stenner (2008/2009), Stephenson estaria sobretudo interessado em explorar as fundações empíricas do construtivismo, isto é, em explorar os processos de construção da representação individual da realidade a partir da experiência. Recorreu, assim, à metodologia $\mathrm{Q}$ para investigar $\mathrm{a}$ variedade das construções subjectivas da realidade, procurando extrair configurações interindividuais similares (Q sorts) que lhe permitissem definir factores capazes de categorizar essa diversidade representativa, pelo recurso a análises factoriais interpessoais ( $Q$ factor analyses).

Nas palavras de Stenner (2008/2009) a metodologia Q é, então, uma "metodologia construtivista arquetípica" (p. 47), já que se refere sempre ao

5 Para uma revisão histórica mais completa consultar Block (1961), Brown (1980) e Smith (2001). 
estudo dos processos intersubjectivos de construção da realidade na interacção com o meio.

A formulação teórica fundacional de Stephenson (1986) para a metodologia Q como método de estudo da complexidade fenomenológica da experiência humana, é coerente com a sua crítica relativamente à orientação empírico-positivista dos paradigmas dominantes da psicologia - behaviorismo e cognitivismo - os quais, segundo ele, estariam presos a um determinismo linear causa-efeito característico da corrente positivista do pensamento científico aplicado à psicologia.

Ainda de um ponto de vista epistemológico, Febbraro (1995) considera que a metodologia $Q$ reflectia, na sua origem, uma orientação individualista, fundada na perspectiva subjectiva/interna da percepção da realidade no estudo empírico dos fenómenos sociais, por oposição às correntes "objectivistas" (empírico-positivistas) da psicologia behaviorista dos anos 1930-1960 .

De facto, a substituição do estudo pearsoniano das "correlações entre testes" pelo estudo de "correlações entre pessoas" (Stephenson, 1935) está na origem, pelo menos tácita, da valorização das abordagens empíricas centradas na pessoa relativamente às abordagens orientadas para o estudo de variáveis no estudo da interacção dos factores de risco e de protecção em relação às patologias mentais e às perturbações do comportamento de crianças, adolescentes e adultos, em particular entre os psicólogos e investigadores inscritos no modelo ecológico-transaccional da psicologia desenvolvimental (Cicchetti \& Rogosch, 1999).

Contudo, o contributo mais interessante de Febbraro (1995) relativamente ao posicionamento epistemológico da metodologia $Q$, a partir da colocação originária como método de investigação da subjectividade individual na ciência behaviorista, é a hipótese segundo a qual esta metodologia poderá ser utilizada como instrumento retórico para "legitimar a ciência como meio de obter conhecimentos sistemáticos, objectivos, válidos, sobre o mundo", pelo que, tem sido usada para uma variedade de agendas (científicas) em variadas circunstâncias" (Febbraro, 1995, p. 149).

Por estas razões, a metodologia Q é considerada por diferentes autores que contribuíram para o seu desenvolvimento e aperfeiçoamento (Brown, 1993, 1996; Stephenson, 1953; van Exel \& Graaf, 2005) como particularmente apropriada à tradução empírica da diversidade de ideias, perspectivas, crenças e fenómenos de natureza subjectiva, que caracterizam o estudo dos estados mentais e das manifestações comportamentais dos sujeitos humanos.

De entre a grande diversidade de temas psicossociológicos que têm sido objecto de investigação utilizando a Metodologia Q, podem ser destacados: os estilos afectivos e cognitivos (e.g. Rayner \& Warner, 2003; Samuels \& Fetzer, 2008), os sistemas de crenças (e.g. Shinebourne \& 
Adams, 2007), os comportamentos e as emoções (e.g. Whyte, Constantopoulos, \& Bevans, 1982; Watts \& Stenner, 2005b), o estudo da personalidade (e.g. Block, 1961), a avaliação sobre processos pedagógicos (e.g. Sheridan, \& Schuster, 2001) ou sobre processo e resultados terapêuticos (e.g. Ablon \& Jones, 2002; Serralta, Nunes, \& Eizirik, 2007), o estudo de representações (e.g. Snelling, 1999), percepções e atitudes (e.g. Santos \& Schor, 2003; Lai et al., 2007).

A metodologia $\mathrm{Q}$ fornece, mais precisamente, uma base para o estudo de questões com características subjectivas, como os sistemas de crenças, valores e atitudes individuais (Brown, 1993; Mckeown \& Thomas, 1988), expressas pelos pontos de vista e opiniões pessoais.

Em termos operacionais, os sujeitos são colocados perante um conjunto de afirmações, que constituem a amostra designada $Q$-set e que envolve uma selecção de itens (afirmações) extraídos de um universo especializado de opiniões e de posições possíveis relativamente à questão ou tema em estudo (Hurd \& Bown, 2004-5). Os participantes que, no seu conjunto, definem o $P$-set, deverão ordenar as afirmações de acordo com o seu ponto de vista. A ordenação obtida é então sujeita a análise factorial.

Stephenson (1935) chegou a apresentar a metodologia Q como uma inversão da análise factorial convencional, uma vez que Q correlaciona pessoas em vez de testes ${ }^{6}$. A correlação entre os perfis pessoais (Brown, 1993) indica, então, semelhantes pontos de vista. Ao correlacionar pessoas, a análise do factor Q fornece semelhanças e/ou diferenças de visões sobre um assunto, e, deste modo, os factores resultantes representam clusters mais operacionalizáveis da subjectividade de temas como atitudes, valores e crenças (Brown, 2002).

Numa investigação que recorra a esta metodologia, sujeitos e variáveis são invertidos relativamente às metodologias convencionais (Webler, Danielson, \& Tuler, 2009), dado que os "sujeitos" do estudo são as diferentes afirmações encontradas sobre o assunto em análise e as variáveis são as pessoas (participantes), ou, mais concretamente, as ordenações ( $Q$-sorts) por eles realizadas de acordo com a sua opinião ou entendimento. Para serem ordenadas, as afirmações compõem um todo e não partes separadas, reconhecendo-se neste procedimento que o método recorre também a referências da teoria Gestalt (Watts \& Stenner, 2005a).

Uma premissa fundamental da metodologia Q, conforme já referido, é que a subjectividade é comunicável (Stephenson, 1953, cit in Watts \&

\footnotetext{
${ }^{6}$ De acordo com van Exel e Graaf (2005), Stephenson referia-se ao facto de, contrariamente ao modo convencional, no qual se passa um número reduzido de testes a um grande número de pessoas, na metodologia $\mathrm{Q}$ se passa a um número reduzido de pessoas um número elevado de itens/testes para responder.
} 
Stenner, 2005a; Stephenson, 1963), e que pode ser analisada de forma sistemática, como se de uma unidade comportamental determinada se tratasse. O conjunto de itens resultante da análise efectuada é, então, classificado de acordo com o esquema de referências de cada participante, o que maximiza a comunicação das perspectivas individuais (Bigras \& Dessen, 2002). Deste modo, é o participante no estudo que decide sobre a importância de cada item pela sua organização/ordenação no conjunto apresentado, sendo esta ordenação comparada com todas as outras. A metodologia Q fornece itens (afirmações) que podem ser ordenados e classificados em categorias, de acordo com o significado subjectivo dado por cada sujeito relativamente ao tema em questão. Em rigor, o que acontece é que esta ordenação e classificação em categorias permite uma base para análise estatística de dados, nomeadamente a análise factorial que facilita a organização e a comunicação da questão em estudo (Stephenson, 1953, cit in Watts \& Stenner, 2005a).

Os resultados encontrados num estudo com a metodologia $Q$ podem então ser usados para descrever uma "população de pontos de vista", e não uma "população de pessoas"7 (Risdon, Eccleston, Crombez, \& McCracken, 2003), pelo que, o método também não requer um grande número de sujei$\operatorname{tos}^{8}$ (conforme exposto, um número significativo de itens ou afirmações é apresentado a um número reduzido de participantes). O entendimento dos autores é de que a informação se repete (Brown, 1996, 2009; van Exel \& Graaf, 2005) após um certo número de sujeitos.

Dado que na metodologia $\mathrm{Q}$ se usa uma amostra pequena, os resultados obtidos têm sido por vezes criticados relativamente à sua validade empírica e, consequentemente, à possibilidade de generalização. No entanto, e de acordo com Brown (1980) em resposta a esta crítica, uma vez que não existem critérios externos para definir o ponto de vista pessoal esta questão não é aqui aplicável. Com efeito, ainda segundo o mesmo autor (Brown, 1980, 1996, 2009) existe um número limitado de perspectivas sobre cada tema. Deste modo uma amostra bem estruturada do ponto de vista da diversidade e abrangência do tema, recolhida de um leque de opiniões diversas mas especializadas, irá revelar essas perspectivas independentemente do tamanho da amostra. Os autores crêem que a partir de determinado número de afirmações (em regra algumas dezenas) as perspectivas se repetem não introduzindo novas ideias.

${ }^{7}$ Como os defensores desta metodologia gostam de referir em oposição à chamada "Metodologia R" (r de Pearson).

${ }^{8}$ Apesar de não haver total consonância entre os diferentes autores, os estudos mais recentes (Webler et al., 2009) apontam para uma proporção de 1:3, ou seja, um participante cada três afirmações. 
Por outro lado, Brouwer (1999) considera que o facto de as questões pertencentes a um mesmo domínio não serem analisadas de forma distinta dos itens, mas sim como um todo e em função da sua coerência mútua para o inquirido (teoria Gestalt subjacente) constitui uma das vantagens do método. Deste modo, é possível identificar perfis ou padrões partilhados por várias pessoas sem ser necessário recorrer a amostras numerosas.

Tendo em vista os inquéritos na população geral, a validade estatística não é a maior preocupação para esta metodologia, uma vez que os resultados de um estudo Q são as diferentes opiniões (subjectivas) sobre o tema que está a ser operacionalizado, e não a percentagem da população que adere a cada uma dessas opiniões.

Finalmente, dado que a metodologia $\mathrm{Q}$ integra procedimentos qualitativos e quantitativos, acaba por funcionar como uma ponte entre as metodologias tradicionais (Rhoads et al., 2004-5), muitas vezes consideradas antagónicas pelos investigadores mais cépticos. Ou seja, significa que as metodologias qualitativa e quantitativa, se podem ainda enriquecer e complementar mutuamente.

A título de exemplo, referir-nos-emos a um trabalho orientado para o estudo das atitudes dos terapeutas relativamente a pacientes sofrendo de perturbações ligadas ao abuso e dependência de drogas (Shinebourne\& Adams, 2007). Neste estudo, os autores propuseram-se um duplo objectivo: 1) compreender as atitudes, crenças e experiências dos terapeutas no trabalho com pacientes com problemas de dependência; 2) explorar a adequação da metodologia Q para esta investigação e para a investigação qualitativa em psicoterapia. Partindo da experiência pessoal dos autores, da discussão com terapeutas experientes na área das dependências e da revisão da literatura, reuniram 60 afirmações que constituíram o "Q-set". Os 13 clínicos participantes foram seleccionados de forma a representarem diferentes orientações teóricas. As afirmações foram colocadas em cartões numerados e apresentadas aos respondentes, tendo-lhes sido solicitado que colocassem os cartões em três grupos conforme tivessem uma posição concordante, discordante ou neutra em relação a cada afirmação. De seguida deveriam ordenar os cartões num continuum de "discordo" (-6) a "concordo" $(+6)$, idêntico aos das escalas tipo Likert (Q-sort). Relativamente ao último grupo de questões os participantes foram, então, instruídos para seleccionar as afirmações com as quais concordavam mais, seguidas daquelas com que concordavam um pouco menos, e assim sucessivamente. O mesmo processo foi repetido com os outros grupos de cartões até todos estarem ordenados e ter sido registado o número de afirmações seriadas numa grelha previamente elaborada. Os participantes puderam ainda comentar as afirmações, ou responder a algumas questões complementares, que foram objecto de análise posterior. Para identificar padrões de semelhança e divergência, foi utilizado o programa estatís- 
tico PCQ - Analysis Software for Q Technique (Stricklin \& Almeida, 2001), que permite correlacionar as ordenações de cada participante entre si. Após um conjunto de procedimentos estatísticos, foram identificados quatro factores: aceitação, desafio, ambivalência e doença. Estes factores foram descritos e interpretados com base na classificação atribuída a cada afirmação e na matriz factorial, em conjunto com os comentários dos respondentes.

Por fim, e relativamente aos objectivos do estudo, os autores apuraram que cada factor mostrou ser um construto multifacetado, não correspondendo a uma única teoria ou orientação terapêutica. Concluíram, então, que os resultados sugeriam uma necessidade de os terapeutas reflectirem sobre as suas crenças relativamente à dependência, bem como à necessidade de supervisão. Apesar de se tratar de um estudo-piloto sobre a questão em causa, Shinebourne e Adams (2007) concluíram que o estudo mostra a capacidade da metodologia Q para identificar semelhanças e diversidades de pontos de vista que não correspondem a conceptualizações estabelecidas a priori e valorizaram a capacidade da metodologia Q para combinar investigação fenomenológica e técnicas estatísticas.

\section{Procedimentos da Metodologia Q}

A realização de um estudo com esta metodologia envolve fundamentalmente cinco passos ${ }^{9}$ (Brown, 1980, 1993, 2009; Danielson, 2009a, 2009b; Webler, et al., 2009):

1 - definição do concourse (curso da comunicação)

2 - desenvolvimento da amostra Q ( $Q$ sample)

3 - selecção do $P$ set

4 -Q-sorting

5 - análise e interpretação

${ }^{9}$ Ou ainda, de forma reduzida, três passos: primeiro é desenvolvido um conjunto de afirmações, de seguida é solicitado aos participantes que ordenem essas afirmações ao longo de um continuum de preferência, e por fim os dados são analisados e interpretados (Brown, 1993). 


\section{1 - Definição do concourse ${ }^{10}$ (curso da comunicação)}

Concourse diz respeito à confluência da comunicação em torno de um tema (o que, em termos de análise da comunicação verbal, é distinto da ordem do discurso). Trata-se da recolha de todas as afirmações que os participantes podem fazer sobre o tema em questão. Esta recolha deverá conter todos os aspectos relevantes de todos os "discursos" recolhidos, cabendo ao investigador constituir uma amostra representativa destas afirmações ou depoimentos (Brown, 1993). O concourse verbal ou escrito ${ }^{11}$ pode ser obtido, entre outros procedimentos, através de entrevistas, observação participante, literatura científica ou documental. O material recolhido representa os pareceres e opiniões de profissionais, de investigadores ou de outros actores credíveis sobre o tema em apreço e constitui a matéria-prima para a metodologia Q (Brown, 1993).

\section{2 - Desenvolvimento da amostra $Q$}

A partir do concourse é desenhado um conjunto inicial de afirmações que é apresentado aos participantes - a chamada amostra Q $(Q$ set ou $Q$ sample). A selecção desta amostra é de importância crucial, sendo tida como uma arte, mais do que uma ciência, de acordo com Brown (1980). O investigador usa uma determinada estrutura (ou construção) para seleccionar uma amostra representativa de todas as comunicações ${ }^{12}$ (concourse). Esta estrutura pode emergir de uma análise mais aprofundada das declarações recolhidas, ou pode ser sujeita a uma determinada teoria.

Independentemente da estrutura usada, o investigador tem de seleccionar a amostra de forma suficientemente alargada, de modo a que esta seja representativa.

Especificando, e conforme já foi referido, as afirmações são inicialmente seleccionadas a partir de uma revisão da literatura sobre a questão em estudo, e de entrevistas ao um painel especialistas que opinam sobre o tema. Numa entrevista ou consulta de opinião, o investigador pode partir de uma questão inicial. Por exemplo, em estudos sobre a personalidade criativa ou

10 Palavra sem tradução correctamente aplicável neste contexto em português, mas que, na língua inglesa e dentro do âmbito da Metodologia Q, é definida como "the initial collection of statements regarding a particular topic of interest" (Brown, 1993).

11 Existem outras possibilidades como objectos e fotografias, mais úteis em estudos noutras áreas.

12 O número de itens da amostra é variável, no entanto o mais frequente é esta ser constituída por 40 a 50 afirmações, até por condicionamentos relativos ao uso dos programas informáticos de apoio (ex. Flash Q). 
sobre o significado de Ego (Block, 1961) poderá perguntar-se respectivamente "o que significa para si personalidade criativa?" e "o que os psiquiatras e psicólogos entendem por força do ego?". Desta fase exploratória, são recolhidas as afirmações mais representativas e que revelam a diversidade de opiniões, em regra de acordo com métodos de análise de conteúdo. Para melhor organização da informação recolhida, podem ainda ser constituídas categorias. Posteriormente é seleccionado um número de afirmações que representam cada uma das categorias. Por exemplo, no caso de oito categorias encontradas, poderão ser seleccionadas 4 ou 5 frases para representar cada categoria, o que perfaz um conjunto de 32 a 40 afirmações, e que constituem a Q sample (Webler, et al., 2009). Diferentes investigadores podem chegar a diferentes amostras $Q$ partindo do mesmo conjunto inicial de afirmações.

De acordo com alguns autores (Thomas \& Baas, 1993) os estudos comparativos realizados e referidos na literatura (em número limitado) apontam no sentido de que diferentes amostras de afirmações, estruturadas de modos diferentes, convergem para as mesmas conclusões.

As afirmações podem inicialmente ser menos objectivas, o que faculta a possibilidade de diferentes interpretações por diferentes participantes, já que serão agrupadas e ordenadas posteriormente, o que permite construir uma definição mais rigorosa do conceito ou do tema em causa.

Importa, contudo, que o material seleccionado no concourse seja representativo do assunto a estudar. Cada afirmação destina-se a ser interpretada no contexto de todas as outras, sendo as informações ordenadas no seu conjunto. Como uma referência indirecta à Psicologia Gestalt, importa aqui, a visão do todo, já que o todo ordenado e relacionado entre si não é o mesmo que a soma das partes ou afirmações. Não compete ao investigador preocupar-se com esta ordenação, pois ela é o objectivo do trabalho dos respondentes e o foco do estudo.

Por último, as afirmações seleccionadas são escritas em cartões aos quais é atribuída uma numeração aleatória, a fim de serem ordenadas de acordo com a instrução dada numa fase posterior - o q-sorting.

\section{3 - Selecção do P set}

O conjunto de pessoas ou participantes que vão ordenar as afirmações (fazer o $Q$-sort) é designado por $P$ set, sendo que a metodologia Q não exige um grande número de participantes (Brown, 1978, 1980). O que é exigido, outrossim, é que os sujeitos sejam em número suficiente para estabelecer a 
existência de um factor ${ }^{13}$ que possa ser comparado com outros. Importa também que assegure a necessária abrangência, a fim de maximizar a confiança de que os principais factores da questão foram identificados. De acordo com alguns autores (Brouwer, 1999; Brown, 1993; Webler et al., 2009), o P set deve ser mais pequeno ${ }^{14}$ do que o $Q$ set.

Os participantes $\mathrm{Q}$ são seleccionados de forma a serem representativos de uma determinada população, tendo em conta a questão em estudo. Por exemplo, se o objecto de estudo for as atitudes terapêuticas em diferentes abordagens teóricas, devem ser contactados terapeutas experientes nas abordagens em estudo, acreditados pelas respectivas entidades/sociedades científicas e distribuídos por características como género, idade e anos de experiência (Block, 1961; Shinebourne \& Adams, 2007; Watts \& Stenner, 2005a).

A selecção tem por objectivo garantir que os sujeitos informantes representam uma larga amplitude de opiniões, sempre no contexto da população-alvo do estudo, e não a distribuição de opiniões ou crenças na população em geral.

Por isso, o $P$ set não é aleatório, mas antes uma amostra estruturada de participantes teoricamente relevantes para a discussão do tema em questão, com opiniões claras e distintas (Brown, 1978).

\section{4-Q-sorting}

De acordo com o procedimento geral (Brown, 1993), o Q set (ou $\mathrm{Q}$ sample) é apresentado aos participantes sob a forma de um conjunto de cartões numerados aleatoriamente, contendo cada um uma afirmação. Ao respondente é pedido para ordenar os cartões de acordo com uma regra, mais precisamente a condição de instrução (ou critério de instrução) que representa normalmente o ponto de vista/opinião sobre o tema em causa. É ainda fornecida uma folha de pontuação, bem como a instrução para a distribuição da ordenação. A pontuação apresenta-se distribuída num continuum cujos extremos são as afirmações "concorda menos" e "concorda mais".

O trabalho de campo tem uma sequência lógica que evolui do seguinte modo: a) primeiro é pedido ao participante que leia cuidadosamente todas as afirmações de modo ficar com uma ideia geral da variedade de opiniões sobre a questão em causa; b) depois, é instruído para que, à medida que for

13 Factor, refere-se a um o conjunto de participantes cujos $Q$-sort foram semelhantes, ou seja, que classificaram as afirmações numa ordem semelhante de preferência, representando cada factor um tipo diferente de opinião

14 Conforme já referido, a regra geral (Webler, et al., 2009) sugere uma proporção entre o $P$ set e o $Q$ set de 1 participante para 3 afirmações. 
lendo, comece a elaborar uma primeira ordenação das afirmações segundo as três categorias (montes de cartões) seguintes: aquelas com que concorda genericamente, aquelas de que discorda e as afirmações relativamente às quais é neutro (fica indeciso ou que lhe suscitam dúvidas); c) o número de afirmações em cada categoria é anotado para verificar o equilíbrio entre concordância e discordância dentro do $Q$ set; d) finalmente, o respondente é convidado a ordenar as afirmações de acordo com a "condição de instrução", colocando-as na folha de pontuação fornecida.

As instruções metodológicas clássicas recomendam que a administração do $Q$ set seja realizada com recurso à entrevista presencial. Todavia, alguns investigadores experimentados nesta metodologia (Van Tubergen \& Olins, 1979), argumentam que os estudos Q podem igualmente ser efectuados por via postal, considerando até que, nestas circunstâncias, conseguem obter resultados mais congruentes do que aqueles que são obtidos através de entrevista presencial.

Mais recentemente, a possibilidade de utilização de recursos informáticos tem conduzido a que boa parte dos estudos sejam conduzidos online ${ }^{15}$ (Santos \& Amaral, 2004). A este propósito, Reber, Kaufman e Cropp (2000), realizaram dois estudos de validação comparando $Q$ sorts baseados em entrevistas presenciais e outros com recurso suporte informático específico para Q-sort (online), tendo concluído que não há diferença significativa entre estes dois métodos de administração no que respeita à validade e fiabilidade.

\section{5 - Análise e interpretação}

A análise estatística tem por objecto os diferentes $Q$-sort, os quais funcionam como variáveis qualitativas que representam as perspectivas individuais dos participantes no estudo. Assim, em primeiro lugar, é calculada a matriz de correlação de todos os Q-sort, a fim de identificar as correlações entre as ordenações obtidas. Seguidamente, procede-se à análise factorial para encontrar o número de agrupamentos de $Q$ sort que correspondem aos factores. Procuram-se idênticas visões de um conceito ou variável (Bryman \& Cramer, 1993), ou seja, grupos que partilham uma descrição similar dessas variáveis (Bigras \& Dessen, 2002). O conjunto de factores encontrado é então rodado (e.g. varimax) para obter o conjunto final de factores (Thomas $\&$ Watson, 2002). Cada factor final resultante, representa um grupo de pontos de vista individuais com elevada correlação entre si e não correlacionados com os outros.

15 Existe actualmente uma considerável variedade de software, quer para a apresentação/administração, quer para análise e interpretação de dados dos $Q$ sort (Thomas \& Watson, 2002; Webler et al., 2009). 
De seguida, e tendo em conta as directrizes mais frequentemente referidas na literatura (Brown, 1978, 1993; van Exel \& Graaf, 2005) sugere-se o cálculo da pontuação dos factores e a diferença de pontuações, sendo que a afirmação que define o factor é aquela que tem a pontuação média ponderada normalizada (Z-score). Com base nas médias ponderadas podem ainda ser conhecidas as afirmações distintivas e as de consenso.

Se os investigadores pretenderem perceber de forma mais detalhada o modo como se distribuem os dados e a sua representatividade numa determinada população, poderão ainda recorrer à utilização combinada com outros instrumentos ou métodos (Danielson, 2009a) ${ }^{16}$.

\section{Conclusão}

Conforme foi referido na introdução, entendemos justificar-se cada vez mais a utilização da Metodologia $\mathrm{Q}$ nas ciências sociais e humanas. $\mathrm{O}$ estudo dos comportamentos humanos, de atitudes, de valores e de crenças, pela sua natureza variável e/ou subjectiva, beneficia com a utilização de métodos de investigação capazes de classificar e ordenar esta informação de modo a clarificá-la.

A metodologia Q proporciona uma forma de integrar a metodologia qualitativa com a quantitativa, que são por vezes consideradas incompatíveis, ou, pelo menos, sem aparente união eficaz, mesmo em estudos multimétodo. Oferece uma possibilidade de complementaridade entre as duas metodologias. Parte de pressupostos e da lógica de investigação qualitativa e é associada a procedimentos de análise quantitativa de dados

Dispõe de ferramentas informáticas próprias (para além das já existentes) quer de administração quer de análise de dados que facilitam a sua utilização, de forma exclusiva, ou em conjunto com outros métodos.

Entendemos, assim, que a metodologia Q é válida no campo das ciências sociais e do comportamento, já que é sensível à variabilidade e à subjectividade particulares aos fenómenos que são objecto de estudo empírico nestas áreas científicas. A metodologia Q permite, mais precisamente, que a estrutura destes fenómenos, por via de regra com características discursivas subjectivas, se torne observável pela operacionalização sistemática que decorre quer da sequência metodológica acima referida, quer da complementaridade de métodos qualitativos e de técnicas estatísticas na construção do conhecimento empírico sobre os fenómenos em estudo.

16 Para mais informação consultar um estudo de Danielson (2009a) em que associa e compara uma análise da narrativa com o Q-sort. Neste trabalho, o autor descreve diferentes técnicas para combinar o Q-sort com outros métodos de pesquisa. 


\section{Referências}

Ablon, S., \& Jones, E. E. (2002). Validity of controlled clinical trials of psychotherapy: Findings rom the NIMH treatment of depression collaborative research program. American Journal of Psychiatry, 159, 775-783.

Bigras, M., \& Dessen, M. A. (2002). O Método Q na avaliação psicológica: utilizando a família como ilustração. Avaliação Psicológica, 2, 119-131.

Block, J. (1961). The Q-sort method in personality assessment and psychiatric research. Springfield: Thomas.

Boros, S., Visu-Petra, L., \& Cheie, L. (2007). A q-sort analysis investigating the social perception of a chronic disease: Between sympathy and stigma. Cognition, Brain, Behavior, 11(2), 347-459.

Brouwer, M. (1999). Q is accounting for tastes. Journal of Advertising Research, 39(2), 35-39.

Brown, S. R. (1978). The importance of factors in Q methodology: statistical and theoretical considerations. Operant Subjectivity, 1(4), 117-124.

Brown, S. R. (1980). Political subjectivity: Applications of $Q$ methodology in political science. New Haven: Yale University Press.

Brown, S. R. (1993). A primer on Q methodology. Operant Subjectivity, 16(3/4), 91-138.

Brown, S. R. (1996). Q Methodology and Qualitative Research. Qualitative Health Research, 6(4), 561-567.

Brown, S. R. (2002). Q technique and questionnaires. Operant Subjectivity, 26(2), 117-126.

Brown, S. R. (2009). Q technique, method, and methodology: Comments on Stentor Danielson's article. Field Methods, 21(3), 238-241.

Bryman, A., \& Cramer, D. (1993). Análise de dados em ciências sociais: Introdução às técnicas utilizando o SPSS. Oeiras, Lisboa: Celta.

Cicchetti, D., Rogosch, F. (1999). Psychopathology as risk for adolescent substance use disorders: adevelopmentalpsychopathology perspective. Journal of Clinical Child \& Adolescent Psychology, 28(3), 355-365.

Danielson, S. (2009a). Q Method and surveys: Three ways to combine Q and R. Field Methods, 21(3), 219-237.

Danielson, S. (2009b). Recognizing common ground: A reply to Steven R. Brown. Field Methods, 21(3), 242-243.

Febbraro, A. (1995). On the epistemology, metatheory, and ideology of Q methodology: a critical analysis. In I. Lubek, van Hezewijk, R., Pheterson, G., \& Tolman, C. (Ed.), Trends and issues in theoretical psychology (pp. 144-150). New York: Springer.

Hurd, R., \& Bown, S. (2004-5). The Future of the Q Methodology Movement. Operant Subjectivity: Journal of the International Society for the Scientific Study of Subjectivity, 28(1/2), 58-75. 
Lai, J.-S., Kupst, M. J., Cella, D., Brown, S. R., Peterman, A., \& Goldman, S. (2007). Using Q-methodology to understand perceived fatigue reported by adolescents with cancer. Psycho-Oncology, 16(5), 437-447.

Mckeown, B., \& Thomas, D. (1988). Q Methodology. Newbury Park: Sage Publications.

Rayner, G., \& Warner, S. (2003). Self-harming behaviour: from lay perceptions to clinical practice. Counselling Psychology Quarterly, 16(4), 305-329.

Reber, B. H., Kaufman, S. E., \& Cropp, F. (2000). Assessing Q-Assessor: A validation study of computer-based Q Sorts versus paper Sorts. Operant Subjectivity, 23(4), 192-209.

Rhoads, J., C., Kinsey, D. F., Popovich, M. N., Focht, W., Stricklin, M., Christman, P., et al. (2004-5). Commentaries on "The Future of the Q Methodology Movement". Operant Subjectivity: Journal of the International Society for the Scientific Study of Subjectivity, 28(1/2), 76-92.

Risdon, A., Eccleston, C., Crombez, G., \& McCracken, L. (2003). How can we learn to live with pain? A Q-methodological analysis of the diverse understandings of acceptance of chronic pain. [doi:10.1016/S0277-9536(02)00043-6]. Social Science \& Medicine, 56(2), 375-386.

Samuels, J. G., \& Fetzer, S. (2008). Development of the Samuels Scale to Rate Pain Management Documentation. [doi: 10.1016/j.pmn.2008.06.004]. Pain Management Nursing, 9(4), 166-170.e164.

Santos, L., \& Amaral, L. (2004, Novembro). Estudos Delphi com Q-Sort sobre a web: a sua utilização em sistemas de informação. Comunicação pessoal apresentada na Conferência da Associação Portuguesa de Sistemas de Informação, actas da $5^{\mathrm{a}}$ conferencia, Lisboa.

Santos, S., \& Schor, N. (2003). Vivências da maternidade na adolescência precoce. Revista Saúde Pública, 37(1), 15-23.

Serralta, F. B., Nunes, M. L. T., \& Eizirik, C. L. (2007). Elaboração da versão em português do Psychotherapy Process Q-Set. Revista de Psiquiatria do Rio Grande do Sul, 29, 44-55.

Sheridan, S., \& Schuster, K. M. (2001). Evaluation of pedagogical quality in early childhood education: A cross-national perspective. Journal of Research in Childhood Education, 16(1), 109-124.

Shinebourne, P., \& Adams, M. (2007). Therapists' understandings and experiences of working with clients with problems of addiction: A pilot study using Q methodology. Counselling \& Psychotherapy Research, 7(4), 211-219.

Smith, N. W. (2001). Current Systems in Psychology: History Theory Research, and Applications. Belmont, CA: Wadsworth/Thomson Learning.

Snelling, S. J. (1999). Women's perspectives on feminism. Psychology of Women Quarterly, 23(2), 247.

Stenner, P. (2008/2009). Q as constructivist methodology. Operant Subjectivity, 32, 46-69.

Stephenson, W. (1935). Correlating persons instead of tests. Character and Personality, 4, 17-24. 
Stephenson, W. (1953). The study of behavior; Q-technique and its methodology. Chicago: The University of Chicago Press.

Stephenson, W. (1963). Independency and Operationism in Q-Sorting. Psychological Record, 13, 269-272.

Stephenson, W. (1986). William James, Niels Bohr and complementarity, I: Concepts. The Psychological Record, 36, 519-527.

Stricklin, M., \& Almeida, J. (2001). PCQ: Analysis software for Qtechnique (rev. ed.) [computer software]. Portland OR, USA: PCQ Software.

Thomas, D. B., \& Baas, L. R. (1993). The issue of generalization in Q-methodology: "reliable schematics" revisited. Operant Subjectivity, 16, 18-36.

Thomas, D. M., \& Watson, R. T. (2002). Q-sorting and MIS research: A primer. Communications of the Association for Information Systems, 8, 141-156.

van Exel, J., \& Graaf, G. (2005). Q-methodology: A sneak preview. Acedido a 20, Julho, 2009 em www.qmethodology.net

Van Tubergen, G. N., \& Olins, R. A. (1979). Mail vs personal interview administration for Q sorts: a comparative study. Operant Subjectivity, 2(2), 51-59 .

Watts, S., \& Stenner, P. (2005a). Doing Q methodology: theory, method and interpretation. Qualitative Research in Psychology, 2(1), 67-91.

Watts, S., \& Stenner, P. (2005b). The subjective experience of partnership love: A Q Methodological study. British Journal of Social Psychology, 44(1), 85-107.

Webler, T., Danielson, S., \& Tuler, S. (2009). Using Q method to reveal social perspectives in environmental research. Greenfield MA: Social and Environmental Research Institute. Acedido a 14, Abril, 2010 em www.serius.org/pubs/Qprimer.pdf.

Whyte, C. R., Constantopoulos, C., \& Bevans, H. G. (1982). Types of countertransference identified by Q-analysis. British Journal of Medical Psychology 55 (Pt 2), 187-201. 